\title{
The Excess Costs of Depression and the Influence of Sociodemographic and Socioeconomic Factors: Results from the German Health Interview and Examination Survey for Adults (DEGS)
}

\author{
Hannah König ${ }^{1}\left[\right.$ ] Alexander Rommel ${ }^{2}$ (I) $\cdot$ Julia Thom $^{2} \cdot$ Christian Schmidt $^{2} \cdot$ Hans-Helmut König ${ }^{1}$ (1) \\ Christian Brettschneider ${ }^{1}\left[\right.$ (i) Alexander Konnopka ${ }^{1}[$
}

Accepted: 4 January 2021 / Published online: 1 February 2021

(C) The Author(s) 2021

\begin{abstract}
Introduction The aim of this study was to estimate excess costs of depression in Germany and to examine the influence of sociodemographic and socioeconomic determinants.

Methods Annual excess costs of depression per patient were estimated for the year 2019 by comparing survey data of individuals with and without self-reported medically diagnosed depression, representative for the German population aged 18-79 years. Differences between individuals with depression $(n=223)$ and without depression $(n=4540)$ were adjusted using entropy balancing. Excess costs were estimated using generalized linear model regression with a gamma distribution and log-link function. We estimated direct (inpatient, outpatient, medication) and indirect (sick leave, early retirement) excess costs. Subgroup analyses by social determinants were conducted for sex, age, socioeconomic status, first-generation or second-generation migrants, partnership, and social support.

Results Total annual excess costs of depression amounted to $€ 5047$ (95\% confidence interval [CI] 3214-6880) per patient. Indirect excess costs amounted to $€ 2835$ (1566-4103) and were higher than direct excess costs (€2212 [1083-3341]). Outpatient (€498), inpatient (€1345), early retirement (€1686), and sick leave (€1149) excess costs were statistically significant, while medication (€370) excess costs were not. Regarding social determinants, total excess costs were highest in the younger age groups ( $€ 7955$ for 18-29-year-olds, $€ 9560$ for 30-44-year-olds), whereas total excess costs were lowest for the oldest age group ( $€ 2168$ for $65+)$ and first-generation or second-generation migrants ( $€ 1820)$.

Conclusions Depression was associated with high excess costs that varied by social determinants. Considerable differences between the socioeconomic and sociodemographic subgroups need further clarification as they point to specific treatment barriers as well as varying treatment needs.
\end{abstract}

Christian Brettschneider and Alexander Konnopka share last authorship.

Supplementary Information The online version contains supplementary material available at https://doi.org/10.1007/s4027 3-021-01000-1.

Hannah König

ha.koenig@uke.de

1 Department of Health Economics and Health Services Research, Hamburg Center for Health Economics, University Medical Center Hamburg-Eppendorf, Martinistraße 52, 20246 Hamburg, Germany

2 Department 2: Epidemiology and Health Monitoring, Robert Koch Institute, Berlin, Germany

\section{Introduction}

Depressive disorders account for the third-highest number of years lived with disability (YLD) worldwide and for 7.5\% of the total disease burden (YLD) in Germany $[1,2]$. The 12 -month prevalence of depression in Germany was estimated to be $9.9 \%$ in females and $4.2 \%$ in males, and for selfreported medically diagnosed depression, it was estimated to be $8.1 \%$ and $3.8 \%$, respectively [3]. Findings from two population-based surveys in Germany showed that the prevalence of depression did not change over time, but mental health outcomes (e.g., days with reduced activity) worsened, indicating that the burden of depression increased for the individual as well as society [4].

In this study, we matched a control group of individuals without depression to a group of individuals with depression 


\section{Key Points for Decision Makers}

The excess costs of individuals with depression vs. individuals without depression were 2.0-times higher for direct and 2.2-times higher for indirect excess costs.

A high share of excess costs accounted for by early retirement indicates that depression has severe consequences, not only in the short term in terms of the number of days spent on sick leave, but also in the long term in the form of permanent productivity losses.

Depending on the subgroup considered, different patterns emerge. As for age, the excess costs of depression were highest among young people (aged 18-29 years), and decreased with increasing age.

and calculated the difference in mean costs between the group of individuals with depression and the control group (excess costs) to estimate the effect of depression on direct and indirect costs, i.e., the average treatment effect on the treated. The findings from a meta-analysis on cost-of-illness (COI) studies of depression showed that research has widely studied the cost of depression, but the proportion of studies reporting excess costs was comparatively small [5]. In the meta-analysis, the ratio of means (RoM) was utilized as an effect measure for the individual study results. The RoM is defined as the relative difference in costs between individuals with depression and individuals without depression [6, 7]. The pooled RoM of direct costs in adults was 2.58 (95\% confidence interval $[\mathrm{CI}] 2.01-3.31$ ), meaning that costs were $158 \%$ higher in individuals with depression than in the control group $[5,8]$. The pooled RoM of indirect costs in adults was 2.28 (95\% CI 1.75-2.98). The meta-analysis revealed an age effect: the relative difference in direct excess costs was highest among adolescent participants and decreased with age, being lowest among oldest ( $\geq 60$ years) participants. The studies on the excess costs of depression that originated in Germany focused on particular subgroups (i.e., older age groups [9-11], anthroposophic care recipients [12], or the members of a specific health insurance fund [13]). Overall, evidence on the excess costs of depression from a societal perspective (i.e., including indirect excess costs) was sparse. Only one German study [12] assessed indirect costs, although depression is well known to be associated with productivity losses [14-16].

Although there are sociodemographic inequalities in the prevalence of depression [17], little is known about the extent to which sociodemographic determinants influence the excess costs of depression. Previous COI studies of depression reporting excess costs have investigated the influence of sociodemographic determinants on overall health care costs without distinguishing between individuals with depression and individuals without depression.

Our primary objective was to estimate the direct and indirect excess costs of depression for adults in Germany using data from the German Health Interview and Examination Survey for Adults (Studie zur Gesundheit Erwachsener in Deutschland, DEGS). The second objective was to investigate whether the association between age and excess costs of depression that was found in the meta-analysis could be replicated. This study further extends current knowledge of the excess costs of depression in Germany, in particular for indirect costs, by using data that are representative of the German population aged 18-79 years. With this, policy makers will get a more precise estimate about the costs attributable to depression for Germany. Reporting excess costs by cost categories could identify resource-intensive health care services used by individuals with depression, information that can be taken into account when developing innovative care approaches (such as shifting inpatient care to outpatient care). The detailed results can provide useful information for future health economic modeling studies. Furthermore, a particular strength of using survey data lies in the possibility to analyze sociodemographic determinants, which are often only present in a rudimentary form in other data sources such as claims data. The excess costs by social subgroups can provide information on different patterns in the utilization of health care services and productivity losses, or potential barriers in the access to care.

We hypothesized that (1) direct and indirect costs are higher in individuals with depression than in individuals without depression, (2) excess costs are highest in the younger age groups and decrease with age, and (3) the excess costs of depression depend on sociodemographic and socioeconomic factors, including age, sex, partnership, social support, socioeconomic status (SES), and first-generation or second-generation migrants.

\section{Methods}

\subsection{Study Design}

The DEGS is part of the health monitoring conducted by the Robert Koch Institute. The design and method of the DEGS have been described elsewhere [18, 19]. The DEGS was conducted from 2008 to 2011 and included interviews, examinations, and tests [20]. Self-administered questionnaires were distributed to participants to obtain information on the physical, psychological, and social aspects of their 
health. Information related to the patient's medical history was obtained by physicians during computer-assisted personal interviews (CAPIs) [18-20]. In total, 8151 people participated in the DEGS, permitting representative crosssectional analysis for people aged 18-79 years [19].

\subsection{Study Sample}

The DEGS collected data on diagnosed depression and depressive symptoms. Diagnosed depression was measured as self-reported medically diagnosed depression in the 12 months prior to the interview. In a CAPI, study participants were asked "Have you ever been diagnosed with depression by a physician or a psychotherapist?" (lifetime); if approved, this was followed by the question "Was the depression present during the last 12 months?" Depressive symptoms during the preceding 2 weeks were measured using the German version of the Patient Health Questionnaire 9 (PHQ-9), wherein a score of $\geq 10$ indicates at least moderate depressive symptoms [21, 22].

As the individuals with depression needed to be composed exclusively of participants with a confirmed diagnosis of depression, only participants with self-reported diagnosed depression were included. Previous studies have shown that costs were also higher when depression was assessed with the PHQ-9 [23-25]. To reduce the risk of including depression-related costs (e.g., as a result of undiagnosed depression or underreporting) in the control group, we did not include participants with self-reported diagnosed depression or current depressive symptoms (i.e., with a PHQ-9 score of $\geq 10$ ). Further, the study sample was restricted to participants without missing values (complete data analysis).

\subsection{Cost Assessment}

We used DEGS data on service utilization and productivity losses to value these outcomes monetarily using average unit costs to estimate annual direct and indirect costs. The recall period on self-reported health service utilization was the 12 months prior to the interview with the exception of medication use (7 days) and any outpatient or inpatient medical rehabilitation (36 months). We estimated weekly medication costs and extrapolated them to annual costs. For medical rehabilitation, only the calendar year preceding the examination year was considered. Thus, the survey period only overlapped with the survey periods of the other variables, but it ensured that the frequency with which medical rehabilitations were considered actually corresponded to 12 months. As no information was provided regarding the duration of medical rehabilitation, we assumed the mean duration of 30 days reported for Germany [26]. A detailed description of the methodology and the findings in relation to utilization of services can be found elsewhere [27]. After costs were allocated to all services, the individual items were summed to provide total costs, which in turn were reported separately in terms of direct and indirect costs. Direct costs were divided into outpatient, inpatient, and medication costs. Outpatient and inpatient costs were further divided into various service providers. For indirect costs, a distinction was made between early retirement and sick leave.

Direct costs were assessed by valuing self-reported health service utilization monetarily. We valued $79 \%$ of costs using standardized unit costs for Germany [28] and medication costs using published medication prices from the German drug catalogue "Rote Liste" [29]. If unit costs were not available, we used data from an internal database of our institute (unpublished material) or the arithmetic mean of all other unit costs within the same cost category (e.g., mean over all outpatient physician specialization, if unit cost for a specific specialization was missed). More details on the unit costs can be found in the Electronic Supplementary Material (see S1 and S2). Indirect costs were assessed as productivity losses using the human capital approach. Productivity losses were valued monetarily using the average gross hourly wage in 2011 for full-time and part-time employees plus the employer's contribution to social insurance [30, 31]. The costs of sick leave were calculated based on the selfreported number of days spent on sick leave and weekly working hours. We estimated costs of early retirement if the study participants stated that they were retired for health reasons. We assumed the average daily working time of fulltime and part-time employees as productivity losses [31], which we valued with the average wage rates $[30,31]$. If the study participants indicated that they were still employed, we subtracted the reported working hours from the assumed productivity losses. The duration of the retirement was unknown, so we have conservatively assumed 105.9 days (half a year without average days off due to holidays or sick leave) of lost productivity to estimate annual costs of early retirement. We accounted for inflation using consumer prices and reported the adjusted costs in 2019 euros [32].

\subsection{Subgroup Analyses}

We additionally estimated excess costs for social subgroups, which we selected based on previous findings on the utilization of various medical services in Germany. Thus, in addition to sociodemographic subgroups such as age and gender, socioeconomic subgroups such as education and income, first-generation or second-generation migrants, and indicators of social ties such as a partner/spouse or a high level of social support were analyzed [27, 33-39]. The influence of age on excess costs of depression was investigated by dividing the sample into four age groups (18-29, 30-44, 45-64, and 65 years and older). SES was calculated as proposed by Lampert and colleagues, which enabled classification into 
low-, medium-, or high-SES groups [40]. Individuals were considered first-generation or second-generation migrants if either the respondent or a parent was born abroad [37, 41]. People living in married or consensual unions were distinguished from those not currently in a relationship (with vs. without a partner). Social support was measured by dividing the 3-Item Oslo Social Support Scale sum score into two categories (low $[<9]$ and moderate/high $[\geq 9]$ ) [42].

\subsection{Statistical Methods}

After the selection of the control group, we adjusted the groups for potential covariates to reduce the risk of confounding in a non-randomized sample. We used entropy balancing (EB) for the group adjustment, as this reweighting method achieves better covariate balance than other common preprocessing methods such as propensity score matching [43]. EB directly assigns a weight to each observation in the control group that matches the treatment group, with minimum deviation from the initial value [43]. After EB, we calculated mean annual costs and corresponding standard errors for all cost categories for both groups, and estimated excess costs of depression using a generalized linear model (GLM) with gamma distribution and a log link [44]. All analyses were weighted using the weights obtained from EB. Thus, no other explanatory variables besides the diagnosis of depression were included in the GLM regression. For the subgroup analyses, EB and the estimation of excess costs were conducted separately for each characteristic of the social subgroups. Statistical significance was defined as $p<0.05$. All statistical analyses were conducted using Stata (version 15). EB was conducted using the Stata package "ebalance" [45].

\subsubsection{Covariates}

We selected social and clinical covariates to adjust the differences in the mean and variance between individuals with depression and the control group. First we selected 45 covariates, of which some were excluded to avoid losing more than $15 \%$ of the observations in the groups due to missing values. In the final model, the socio-demographic covariates balanced for were age, sex, education, income, marital status, community size (rural, small-, mid-sized-, and large town), region (seven Nielsen areas), and physicians' density (physicians per 100,000 inhabitants, categorized into four categories). Clinical covariates concerning health status and risk factors were recognized disability (self-reported official recognition by the pension office [18]), body mass index, contraceptive pill use, use of hearing aid, physical activity (sport hours per week [46]), smoker status (current smoker-even if only occasionally [47]), and use of vision aid (glasses/contact lenses). Furthermore, we balanced for the following comorbidities: chronic inflammatory bowel disease, gastroduodenal ulcer, injury/poisoning, and joint pain (12-month prevalence), as well as arthrosis/degenerative joint disease, bronchial asthma, cancer, diabetes, epilepsy, hepatic cirrhosis, hepatitis, hypertension, kidney failure, migraine, prostatic hyperplasia, rheumatoid arthritis, and thyroid disease (lifetime prevalence), and not specified further diseases (current impairment/treatment). Table 1 provides more details on the included covariates.

Since the number of observations was smaller within the subgroups, we reduced the balancing model by excluding all covariates without statistically significant differences between the groups: education, region, hearing aid, physical activity, bronchial asthma, hepatitis, hepatic cirrhosis, injury/poisoning, kidney failure, prostatic hyperplasia, and rheumatoid arthritis. In addition, the social subgroup that was analyzed was excluded from the related reweighting scheme.

\subsubsection{Sensitivity Analyses}

We conducted several sensitivity analyses to test the robustness of our results in relation to the assessment of productivity loss, the balancing scheme, and the selection of the depression identifier. There are several ways to evaluate productivity loss. Therefore, we tested whether using specific wage rates with respect to employment status and sociodemographic characteristics instead of average wages would change the results in relation to indirect excess costs. Second, we calculated unadjusted excess costs to test the influence of the adjustment on the results. Third, we used both adjusted and unadjusted analyses to test whether the results differed if the individuals with depression comprised those participants with an acute episode of depression, i.e., at least moderate current depressive symptoms based on the PHQ-9.

\section{Results}

\subsection{Sample Characteristics}

The final study sample consisted of 223 individuals with depression and 4540 individuals without depression. Some observations had to be excluded from the analysis for various reasons, including missing data in relation to costs, depression status, or sociodemographic or clinical variables, and the need to exclude participants with depressive symptoms from the control group (see Table S3 in the Electronic Supplementary Material). As shown in Table 1, the sample characteristics of all predefined covariates were balanced between individuals with depression and individuals without depression using EB. After EB, the average age of the participants was 52 years and $68.2 \%$ of the individuals with 
Table 1 Pre- and post-weighting results using entropy balancing

\begin{tabular}{|c|c|c|c|c|}
\hline Weighting covariates & Details & $\begin{array}{l}\text { Individuals } \\
\text { with depression } \\
(n=223)\end{array}$ & $\begin{array}{l}\text { Unmatched } \\
\text { control group } \\
(n=4540)\end{array}$ & $\begin{array}{l}\text { Matched } \\
\text { control group } \\
\left(n=223^{\mathrm{a}}\right)\end{array}$ \\
\hline \multicolumn{5}{|l|}{ Sociodemographic and socioeconomic covariates } \\
\hline Age (mean) & Mean & 51.7 & 49.5 & 51.6 \\
\hline Sex $(\%$ female $)$ & Male $=0$, female $=1$ & 68.2 & 48.9 & 68.0 \\
\hline Education level (\%) & CASMIN classification & & & \\
\hline Low & & 32.7 & 28.5 & 32.8 \\
\hline Middle & & 50.7 & 50.9 & 50.6 \\
\hline High & & 16.6 & 20.6 & 16.6 \\
\hline Income $(€)$ & Mean (equivalent income) & 1408.6 & 1558.6 & 1406.2 \\
\hline \multicolumn{5}{|l|}{ Marital status (\%) } \\
\hline Married, living with partner & & 52.5 & 65.0 & 52.5 \\
\hline Married, separated from partner & & 2.2 & 1.4 & 2.2 \\
\hline Single & & 24.2 & 23.8 & 24.2 \\
\hline Divorced & & 12.1 & 5.5 & 12.1 \\
\hline Widowed & & 9.0 & 4.3 & 9.0 \\
\hline \multicolumn{5}{|l|}{ Community size (\%) } \\
\hline Rural & Inhabitants: $<5000$ & 13.9 & 18.4 & 14.0 \\
\hline Small town & Inhabitants: 5000 to $<20,000$ & 20.6 & 25.6 & 20.6 \\
\hline Mid-sized town & Inhabitants: 20,000 to $<100,000$ & 27.4 & 29.1 & 27.3 \\
\hline Large town & Inhabitants: $\geq 100,000$ & 38.1 & 26.9 & 38.1 \\
\hline Region (\%) & Nielsen areas & & & \\
\hline North-West & & 13.9 & 12.6 & 13.9 \\
\hline North Rhine-Westphalia & & 18.8 & 18.5 & 18.8 \\
\hline Central & & 16.1 & 12.1 & 16.1 \\
\hline East (North) & & 16.1 & 17.4 & 16.1 \\
\hline East (South) & & 10.8 & 14.4 & 10.8 \\
\hline Bavaria & & 13.0 & 12.9 & 13.0 \\
\hline Baden-Wuerttemberg & & 11.2 & 12.0 & 11.2 \\
\hline Physician density (\%) & Physicians per 100,000 inhabitants & & & \\
\hline Approximately 124 & & 12.6 & 19.0 & 12.7 \\
\hline Approximately 144 & & 35.9 & 38.7 & 35.8 \\
\hline Approximately 149 & & 13.5 & 15.9 & 13.5 \\
\hline Approximately 233 & & 38.1 & 26.5 & 38.1 \\
\hline \multicolumn{5}{|l|}{ Clinical covariates } \\
\hline \multicolumn{5}{|l|}{ Health status and risk factors } \\
\hline Recognized disability (\%) & No $=0$, yes $=1$ & 29.2 & 12.1 & 29.1 \\
\hline Body-mass-index (mean) & $\mathrm{kg} / \mathrm{m}^{2}$ & 28.0 & 26.7 & 27.9 \\
\hline Contraceptive pill use (\%) & No $=0$, yes $=1$ & 56.5 & 38.5 & 56.4 \\
\hline Hearing aid (\%) & No $=0$, yes $=1$ & 4.9 & 3.3 & 4.9 \\
\hline Physical activity (\%) & $\begin{array}{l}4 \text { categories (sporting hours per week in the } \\
\text { preceding } 3 \text { months) }\end{array}$ & 63.2 & 69.8 & 63.2 \\
\hline Smoker status (\%) & $\begin{array}{l}\text { No }=0, \text { yes }=1 \text { (including occasional smok- } \\
\text { ers) }\end{array}$ & 32.3 & 25.5 & 32.3 \\
\hline Vision aid (\%) & No $=0$, yes $=1$ & 82.5 & 74.6 & 82.4 \\
\hline \multicolumn{5}{|l|}{ Comorbidities } \\
\hline Arthrosis/degenerative joint disease (\%) & No $=0$, yes $=1$ (lifetime prevalence) & 34.1 & 20.1 & 34.0 \\
\hline Bronchial asthma (\%) & No $=0$, yes $=1$ (lifetime prevalence) & 9.9 & 7.4 & 9.9 \\
\hline Cancer $(\%)$ & No $=0$, yes $=1$ (lifetime prevalence) & 10.3 & 6.3 & 10.3 \\
\hline $\begin{array}{l}\text { Chronic inflammatory bowel disease } \\
(\%)\end{array}$ & No $=0$, yes $=1(12$-month prevalence $)$ & 3.1 & 0.3 & 3.1 \\
\hline
\end{tabular}


Table 1 (continued)

\begin{tabular}{|c|c|c|c|c|}
\hline Weighting covariates & Details & $\begin{array}{l}\text { Individuals } \\
\text { with depression } \\
(n=223)\end{array}$ & $\begin{array}{l}\text { Unmatched } \\
\text { control group } \\
(n=4540)\end{array}$ & $\begin{array}{l}\text { Matched } \\
\text { control group } \\
\left(n=223^{\mathrm{a}}\right)\end{array}$ \\
\hline Diabetes (\%) & No $=0$, yes $=1$ (lifetime prevalence) & 13.0 & 6.8 & 13.0 \\
\hline Epilepsy (\%) & No $=0$, yes $=1$ (lifetime prevalence) & 3.1 & 1.0 & 3.1 \\
\hline Gastroduodenal ulcer (\%) & No $=0$, yes $=1(12$-month prevalence $)$ & 1.8 & 0.4 & 1.8 \\
\hline Hepatic cirrhosis (\%) & No $=0$, yes $=1$ (lifetime prevalence $)$ & 0.9 & 0.2 & 0.9 \\
\hline Hepatitis (\%) & No $=0$, yes $=1$ (lifetime prevalence) & 6.7 & 5.8 & 6.7 \\
\hline Hypertension (\%) & No $=0$, yes $=1$ (lifetime prevalence $)$ & 47.1 & 36.2 & 47.0 \\
\hline Injury/poisoning (\%) & No $=0$, yes $=1(12$-month prevalence $)$ & 10.8 & 10.4 & 10.8 \\
\hline Joint pain $(\%)$ & No $=0$, yes $=1(12$-month prevalence $)$ & 77.6 & 53.5 & 77.5 \\
\hline Kidney failure (\%) & No $=0$, yes $=1$ (lifetime prevalence $)$ & 2.2 & 1.5 & 2.2 \\
\hline Migraine (\%) & No $=0$, yes $=1$ (lifetime prevalence $)$ & 23.3 & 10.1 & 23.3 \\
\hline Prostatic hyperplasia (\%) & No $=0$, yes $=1$ (lifetime prevalence) & 7.6 & 7.9 & 7.6 \\
\hline Rheumatoid arthritis (\%) & No $=0$, yes $=1$ (lifetime prevalence) & 4.5 & 2.3 & 4.5 \\
\hline Thyroid disease (\%) & No $=0$, yes $=1$ (lifetime prevalence $)$ & 33.2 & 23.1 & 33.1 \\
\hline Not specified other diseases (\%) & $\begin{array}{l}\text { No }=0, \text { yes }=1 \text { (current impairment/treat- } \\
\text { ment) }\end{array}$ & 53.8 & 38.0 & 53.7 \\
\hline
\end{tabular}

CASMIN Comparative Analysis of Social Mobility in Industrial Nations

${ }^{\text {a }} N=4540$ observations in the control group were downweighted in order to match the group of individuals with depression

depression and $68.0 \%$ of those without depression were female.

\subsection{Excess Costs}

Total costs were estimated as $€ 9539$ in individuals with depression and $€ 4492$ in individuals without depression. Thus, excess costs amounted to $€ 5047$ (95\% CI 3214-6880). The share of direct and indirect excess costs was €2212 (95\% CI 1083-3341) and €2835 (95\% CI 1566-4103), respectively. All results were statistically significant. Regarding direct cost categories, the differences between the groups were statistically significant for both outpatient costs (€498 [95\% CI 360-636]) and inpatient costs ( $€ 1345$ [95\% CI 446-2244]). Medication excess costs ( $€ 370$ [95\% CI - 241-980]) were not statistically significant. The main contributor to outpatient excess costs was psychotherapist costs (€317), followed by psychiatrist/ neurologist costs $(€ 103)$. Expressed as the relative difference in costs, psychotherapist and psychiatrist/neurologist costs were around 16- and 12-times higher, respectively, in individuals with depression than in individuals without depression. Inpatient excess costs were mainly caused by hospital visits $(€ 1285)$. The indirect cost categories were statistically significant, with a higher share of indirect excess costs attributable to early retirement $(€ 1686)$ than that attributable to sick leave ( $€ 1149)$. See Fig. 1, Table 2, and Supplementary Table S4 (see the Electronic Supplementary Material) for detailed information.

\subsection{Social Subgroups}

Across all determinants, total excess costs ranged from $€ 1820$ (for first-generation or second-generation migrants) to $€ 9560$ (for 30-44-year-olds). Direct excess costs ranged from $€ 1174$ (for first-generation or second-generation migrants) to $€ 4819$ (for 30-44-year-olds), and indirect excess costs ranged from $€ 68$ (for people aged 65 years and older) to $€ 4741$ (for 30-44-year-olds). The comparison of costs within each category (e.g., female) of the social subgroups provides information on which categories have significant excess costs. Total excess costs were statistically significant for females, all age groups except for those aged $65+$ years, individuals with and without partners, all SES groups, individuals with moderate/high social support, and individuals that were not first-generation or second-generation migrants. After dividing total excess costs into direct and indirect excess costs, the results were no longer statistically significant for the high SES group. In addition, indirect excess costs were no longer statistically significant for people aged 18-20 years, without a partner or of low SES. More details are provided in the Electronic Supplementary Material, Table S5.

Conversely, a comparison between the categories of each subgroup revealed the extent to which the categories (e.g., females and males) differ with regard to excess costs, as indicated by the RoM. Although the CIs for the social determinants were wide and overlapped between subgroups, several tendencies can be seen (see Fig. 2a-c). However, caution 
Fig. 1 Estimated mean costs of each cost category (in euros, 2019)

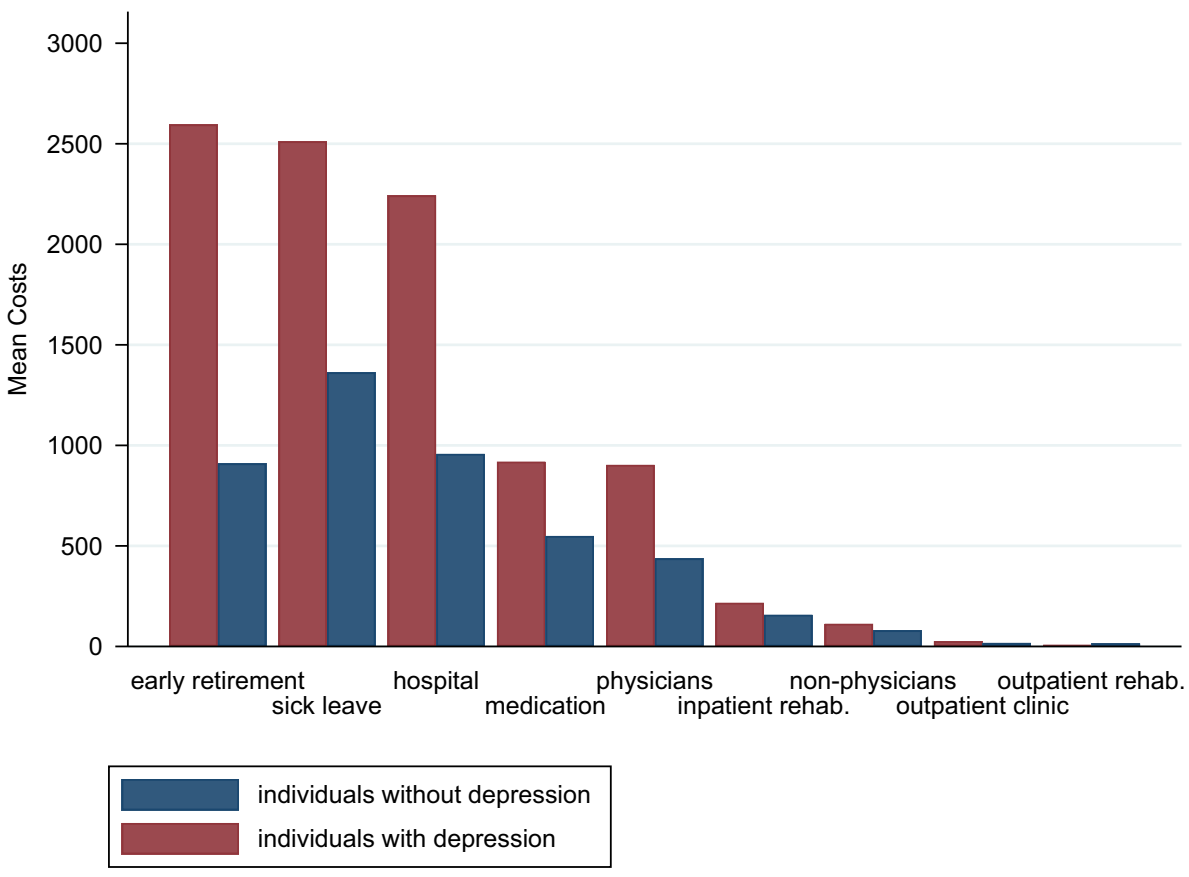

should be exercised when interpreting the results. Younger age was related to higher direct excess costs $(\mathrm{RoM}=4.8$ for 18-29-year-olds and RoM = 4.7 for 30-44-year-olds), which declined with age ( $R=M=1.6$ for 45-64-year-olds and 65+-years-olds). This pattern also appeared in relation to indirect costs for the 18-29 (RoM = 6.1), 30-44 (RoM $=3.0)$, and 45-64 (RoM = 1.7) age groups. We also calculated indirect excess costs for the 65+ age group (RoM $=3.7$ ), although participants at that age tend not to work anymore, as can be seen from the low absolute indirect costs in individuals with depression (€94) and individuals without depression (€26). When considering excess costs by gender, females had higher excess costs than males. Not being in a relationship was correlated with higher direct excess costs, but lower indirect excess costs. The lack of a supportive social network was correlated with lower excess costs. The direct excess costs were higher for individuals with low SES than for individuals with medium or high SES, while the indirect excess costs were not. First-generation or secondgeneration migrants incurred lower excess costs than for their peers.

\subsection{Sensitivity Analyses}

The results of the sensitivity analyses are shown in the Electronic Supplementary Material, Table S6. When productivity loss was assessed using specific wage rates, absolute indirect excess costs were slightly lower than in the main analysis ( $€ 2538$ vs. $€ 2835$; RoM $=2.1$ vs. RoM = 2.2). Contrary to the main analysis, unadjusted excess costs were in general higher and more likely to be statistically significant.
For example, the total excess costs of depression were 2.1times higher in the main analysis and 3.5-times higher in the unweighted analysis. When depression was assessed using the PHQ-9, the excess costs of individuals with an acute episode of depression (i.e., PHQ-9 $\geq 10$ ) also increased relative to non-depressed individuals ( $\mathrm{RoM}=2.0$ ). However, excess costs were generally lower than those of individuals with diagnosed depression, except for the direct cost categories outpatient clinics, general practitioner, other physicians, and outpatient medical rehabilitation.

\section{Discussion}

The purpose of this study was to calculate the direct and indirect excess costs of adults reporting a medical diagnosis of depression using DEGS data and to assess the impact of social determinants on excess costs.

The first hypothesis, that direct and indirect costs are higher in individuals with depression, was supported. Indirect excess costs accounted for approximately $56 \%$ of total excess costs, indicating that the assessment of indirect costs is important from a societal perspective, as a reduction in the labor force directly affects the productivity of a country. In our analysis, the estimated RoM was 2.0 (1.5-2.6) for direct and 2.2 (1.7-3.0) for indirect excess costs, which was to some extent lower than that for the pooled results in the meta-analysis [5]. A possible explanation for the relatively small difference in costs between the groups might be that only unadjusted costs were pooled in the meta-analysis, while we conducted adjusted analyses. The findings of the 
Table 2 Estimated mean annual excess costs of individuals with medically diagnosed depression (12-month prevalence) compared to individuals without medically diagnosed depression (in Euro, 2019)

\begin{tabular}{|c|c|c|c|c|c|}
\hline \multirow[t]{2}{*}{ Cost category } & \multirow{2}{*}{$\begin{array}{l}\text { Depression }(n=223) \\
\text { Mean }(\mathrm{SE})\end{array}$} & \multirow{2}{*}{$\begin{array}{l}\text { Control }(n=223)^{\mathrm{a}} \\
\text { Mean }(\mathrm{SE})\end{array}$} & \multicolumn{3}{|l|}{ Excess costs } \\
\hline & & & Absolute difference $[95 \% \mathrm{CI}]$ & $P$ value & $\begin{array}{l}\text { Relative } \\
\text { difference }^{b} \\
{[95 \% \mathrm{CI}]}\end{array}$ \\
\hline Total costs & $9539(890)$ & $4492(287)$ & 5047 [3214-6880] & $<0.001$ & $2.1[1.7-2.7]$ \\
\hline Direct costs & $4430(557)$ & $2218(145)$ & $2212[1083-3341]$ & $<0.001$ & $2.0[1.5-2.6]$ \\
\hline Outpatient costs & $1050(68)$ & $552(18)$ & 498 [360-636] & $<0.001$ & $1.9[1.6-2.2]$ \\
\hline Physicians & $903(61)$ & $438(13)$ & $465[342-588]$ & $<0.001$ & $2.1[1.8-2.4]$ \\
\hline Psychiatrist/neurologist & $112(17)$ & $10(2)$ & $103[69-136]$ & $<0.001$ & $11.5[7.5-7.7]$ \\
\hline Psychotherapist & $338(49)$ & $21(5)$ & 317 [219-414] & $<0.001$ & $16.2[9.4-7.9]$ \\
\hline General practitioner & $112(8)$ & $83(4)$ & 30 [13-47] & 0.001 & $1.4[1.2-1.6]$ \\
\hline Other physicians & $341(21)$ & $325(11)$ & $16[-31$ to 63$]$ & 0.501 & $1.0[0.9-1.2]$ \\
\hline Non-physicians & $113(18)$ & $81(7)$ & $32[-6$ to 70$]$ & 0.099 & $1.4[1.0-2.0]$ \\
\hline Outpatient clinic ${ }^{c}$ & $26(9)$ & $18(3)$ & $8[-10$ to 27$]$ & 0.366 & $1.5[0.7-3.0]$ \\
\hline Outpatient rehabilitation & $7(7)$ & $15(3)$ & $-8[-23$ to 7$]$ & 0.308 & $0.5[0.1-3.5]$ \\
\hline Inpatient costs & $2461(443)$ & $1116(120)$ & 1345 [446-2244] & 0.003 & $2.2[1.5-3.3]$ \\
\hline Hospital & $2243(438)$ & $958(113)$ & 1285 [399-2172] & 0.004 & $2.3[1.5-3.7]$ \\
\hline Inpatient rehabilitation & $217(61)$ & $158(30)$ & $59[-74$ to 192$]$ & 0.381 & $1.4[0.7-2.7]$ \\
\hline Medication costs & 919 (306) & $550(59)$ & $370[-241$ to 980$]$ & 0.235 & $1.7[0.8-3.3]$ \\
\hline Indirect costs & $5109(618)$ & $2274(192)$ & 2835 [1566-4103] & $<0.001$ & $2.2[1.7-3.0]$ \\
\hline Early retirement & $2596(483)$ & $911(153)$ & 1686 [694-2678] & 0.001 & $2.9[1.7-4.7]$ \\
\hline Sick leave & $2512(453)$ & $1363(129)$ & 1149 [226-2072] & 0.015 & $1.8[1.2-2.7]$ \\
\hline
\end{tabular}

Weighted covariates using entropy balancing: age, sex, education, income, marital status, community size, region, physicians density, recognized disability, body mass index, contraceptive pill use, use of hearing aids, physical activity, smoker status, use of vision aids, arthrosis/degenerative joint disease, bronchial asthma, cancer, chronic inflammatory bowel disease, diabetes, epilepsy, gastroduodenal ulcer, hepatic cirrhosis, hepatitis, hypertension, injury/poisoning, joint pain, kidney failure, migraine, prostate hyperplasia, rheumatoid arthritis, thyroid disease, and not specified other diseases

$C I$ confidence interval, $S E$ robust standard error

${ }^{\mathrm{a}} N=4540$ observations in the control group were downweighted in order to match the group of individuals with depression

${ }^{\mathrm{b}}$ Exponentiated coefficients and corresponding CIs

${ }^{c}$ Hospital emergency or outpatients department or a medical treatment center without overnight stays

sensitivity analyses support this explanation, as the RoMs of the unadjusted excess costs were at or above the upper bound of the $95 \%$ CIs for the pooled RoM in the meta-analysis. Another reason might be that we used data from a population survey, whereas the data used in the meta-analysis were obtained from various data sources and study samples. When our findings were compared with those of studies with a similar study design, the estimated results were within the expected range for direct costs $(\mathrm{RoM}=1.04-4.81)$ and indirect costs $(\mathrm{RoM}=1.70-3.67)$ [48-55].

In our analysis, the individuals with depression were only those study participants with a self-reported diagnosis of depression. If depression was assessed using standard diagnostic instruments (such as PHQ-9, see sensitivity analyses), the use of health services was measured independently of a health professional's depression diagnosis. Thus, a considerable share of them might be affected by an untreated depressive disorder. Therefore, the sensitivity analyses also reflect the presence of unmet needs, resulting in a lower estimate of direct excess costs than that found in the main analysis.

In relation to the second hypothesis, the younger age groups incurred the highest overall excess costs. The RoM was highest at younger age and decreased with age. One possible explanation for this pattern could be that comorbidities increase with age, resulting in higher baseline costs [56, 57]. For the groups of working age, increasing comorbidities might also be more likely to prompt early retirement, resulting in higher indirect baseline costs. As for sick leave, individuals with depression incurred lower costs with increasing age. However, the baseline costs of 18-29-year-olds were lower than for those in older age and peaked in the age group 30-44 years, resulting in decreasing excess costs. Several studies that reported excess costs of depression showed that costs significantly increased with age [10, 48, 54, 58, 59], but none assessed how excess costs varied with age. We found one study that investigated the influence of age on the 
Fig. 2 a Total excess costs by social subgroups (ratio of means). b Direct excess costs by social subgroups (ratio of means). c Indirect excess costs by social subgroups (ratio of means)

$$
\begin{aligned}
& \text { Sex } \\
& \text { male } \\
& \text { female }
\end{aligned}
$$

\section{Age groups 18-29 years 30-44 years 45-64 years $65+$ years}

Partnership

no partner partner

Socieconomic status (SES) low SES medium SES high SES

Social support

low social support moderate/high social support

First/Second-generation no

yes
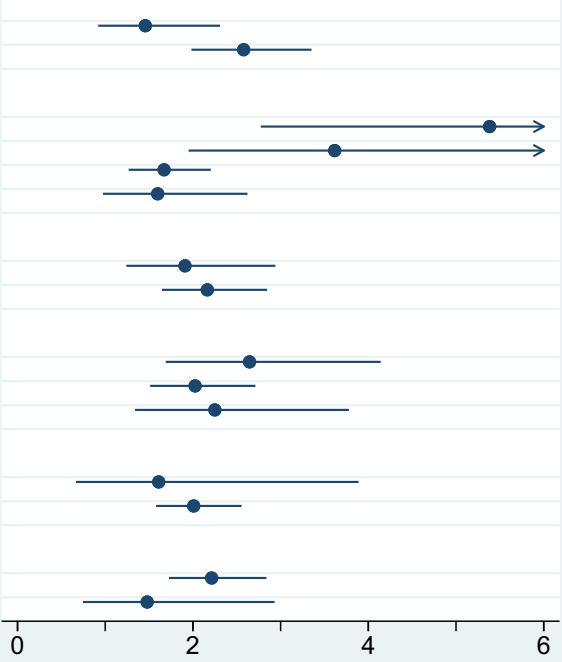

Sex male female

Age groups 18-29 years 30-44 years 45-64 years $65+$ years

Partnership
no partner no partner partner

Socieconomic status (SES) low SES high SES

Social support low social support moderate/high social support

First/Second-generation no yes

Sex male female

\section{Age groups 18-29 years 30-44 years 45-64 years $65+$ years}

Partnership no partner partner

Socieconomic status (SES) low SES

medium SES

high SES

Social support low social support moderate/high social support

First/Second-generation no

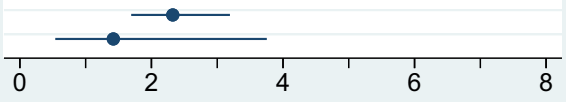


excess costs of depression (18-64 vs. 65+), but the association was not statistically significant [51]. Contrary to our findings, the difference in costs was almost equal for younger and older adults (RoM 2.00 vs. 1.95).

With regard to the third hypothesis, this study is one of the first to analyze the excess costs of depression based on social determinants. Some previous COI studies that have addressed the excess costs of depression have analyzed the influence of social determinants on costs $[9-12,48,54,58$, 60]. However, they all examined the influence of social determinants on overall health care costs, rather than excess costs, and so the results cannot be directly compared with our findings.

In our study, the estimated excess costs were higher among females than among males. This is in line with numerous findings [27, 35, 36, 39]. Previous studies on the use of psychiatric and psychotherapeutic services in Germany have found that service uptake is higher among individuals with a low level of social support and without a steady partner, supporting the finding that these groups incur higher direct costs $[35,39]$. However, the RoM of direct excess costs was lower for individuals with low social support, which needs further investigation. Contrary to this, individuals with low social support or without a steady partnership incurred relatively lower indirect excess costs than their peers. One reason could be that a partnership and a supportive network promotes a more responsible way of dealing with illness in relation to employment. As for SES, the direct costs of the control groups were lower for low SES than for the higher education levels. This supports the findings of studies showing that people with high SES tend to seek specialists in relation to outpatient care, whereas people with low SES tend to seek general practitioners [27, 34]. For individuals with depression, direct costs decreased with higher SES status, resulting in decreasing excess costs. One reason for this might be that lower SES is associated with poorer mental well-being and a higher incidence of mental disorders [3, 61, 62]. Another reason might be that people with a higher education level seek out a more specific therapy, resulting in less health care utilization. Apparently, further research is needed to investigate this relation. It is reasonable to suppose that indirect excess costs were higher with medium and high SES, as greater productivity losses can be expected in relation to people with higher SES. Previous analyses of the utilization of health services by members of migrant communities showed that in Germany, even in relation to medical needs, first-generation or second-generation migrants tend to use services less frequently than their peers $[36,38]$. Possible reasons might be a lack of knowledge about existing health care services or barriers to the treatment (such as language or culture, a lack of intercultural education of persons in the health care sector or the fear of stigmatization) $[37,38,63,64]$. Thus, with regard to excess costs, lower costs could be expected for first-generation or second-generation migrants, which was supported by our analyses.

\subsection{Strengths and Limitations}

This study analyzed the economic burden of depression in Germany using a representative sample of people aged 18-79 years. Using data from a large population-based survey (DEGS) offers several advantages. Depression was assessed by clinically trained interviewers, providing high data quality as compared to analyses based on claims or selfadministered questionnaires. Especially in the case of mental disorders, data from patient surveys can cover more cost categories than claims or medical records [65]. Additionally, DEGS contains measurements of depressive symptoms, which enables sensitivity analysis of individuals having an acute episode of depression. Participants with and without depression were contained in one dataset, and thus a second data source with a potentially different framework was not needed to 'create' the control group. Since information on employment parameters was available, indirect costs as a result of productivity loss could be analyzed. Moreover, many patient characteristics were captured in the DEGS and could be used for the group adjustment. Treating the differences between the groups using EB was an innovative approach that enabled numerous covariates to be adjusted simultaneously. Another strength of this study is that the influence of social determinants on costs was analyzed for both groups, enabling the identification of differences in excess costs between subgroups.

This study also has several limitations. DEGS was conducted from 2008 to 2011. In ambulatory claims data, depression prevalence rose considerably in the last decade, especially in younger age groups [66]. Thus, estimated costs might have changed accordingly. However, in the absence of up-to-date comprehensive survey data, the presented estimation relies on the most recent data for the general population. Further, the results might be biased, because information on utilization of health care services relied on participants' self-reporting (recall bias and reporting bias). We adjusted for observed covariates in the depression and control groups using EB. Therefore, the results might be biased by unobservable variables and unadjusted covariates, like mental disorders other than depression. As for the subgroups, the differences in the excess costs might be to some extent influenced by variations in the level of unmeasured confounding. Furthermore, numerous observations with missing values had to be excluded to facilitate a valid analysis, resulting in small sample sizes when investigating social subgroups. However, when comparing the analyzed sample of individuals with depression with the original sample in DEGS, the sample characteristics with respect to age (52 vs. 53 years), 
sex (68.2\% vs. $69.3 \%$ females), and average PHQ-9 score (9.1 vs. 9.4) remained stable. Self-reported depression diagnosis is a widely used measure of depression in health surveys, even though subject to bias. As validation by standardized depression diagnoses assessed in clinical interview indicates, only $51.8 \%$ of the individuals with self-reported diagnosis of depression met the Composite International Diagnostic Interview (CIDI) criteria of an affective disorder [67]. However, this can be qualified by the fact that there may still be a need for treatment, and that clinical interviews do not assess unspecific depression diagnoses, which are very common in health care [66].

Moreover, severely ill participants are underrepresented in population surveys (e.g., because individuals with severe depression are less likely to participate due to their symptomatology and to be reached because they receive acute treatment in hospitals), increasing the risk of underestimation of costs. This study estimated costs from a societal perspective. However, important cost categories discussed in the literature, such as the costs of informal care, costs of supported accommodation, costs of criminal justice, or suicide-related costs, were not taken into account [68-70].

\subsection{Implications}

Indirect excess costs and inpatient excess costs had the highest share in total excess costs. Innovative care approaches such as integrated care or severity stepped therapy might help reduce hospital stays and days of sick leave, and also meet the needs of those affected. The subgroup analyses showed groups of individuals who receive many health care services, which might indicate a high need for care. In contrast, other subgroups showed comparatively lower excess costs, which could be an indication of existing barriers to care. For example, online interventions could be a helpful tool for individuals who are informed about existing care services, but do not use them due to fear of stigmatization. As for first-generation or second-generation migrants, there might be barriers to the treatment such as language, but also little intercultural education on the part of the health care providers.

\section{Conclusion}

In summary, depression is associated with high direct and indirect excess costs. Our analysis of social determinants showed that the excess costs of depression differed between subgroups, and identified potential barriers such as language or cultural barriers for first-generation or second-generation migrants to the use of health care services. Future COI studies should include a thorough adjustment for group differences to accurately identify disease-specific excess costs.

Acknowledgements We thank Geoff Whyte, MBA, from Edanz Group (http://www.edanzediting.com/ac) for editing a draft of this article.

\section{Declarations}

Funding Open Access funding enabled and organized by Projekt DEAL. The DEGS study is conducted by the Robert Koch Institute as part of the Federal Health Monitoring in Germany on behalf of the Federal Ministry of Health. The ministry finances the Robert Koch Institute and gives substantial funds for the Federal Health Monitoring. Three of the authors are employees of the Robert Koch Institute. The funders had no role in study design, data collection and analysis, decision to publish, or preparation of the manuscript.

Conflict of interest The authors declare that they have no conflict of interest.

Ethics approval The DEGS study protocol was consented with the Federal and State Commissioners for Data Protection and approved by the Charité-Universitätsmedizin Berlin ethics committee in September 2008 (No. EA2/047/08).

Consent to participate In DEGS, participants provided written informed consent prior to the interview and examination. In this study, we did not collect data from patients. Only anonymized data was used.

Consent for publication In DEGS, participants provided written informed consent that the collected anonymized data may be used for research purposes.

Availability of data and material The 'Health Monitoring' Research Data Centre at the Robert Koch Institute (RKI) is accredited by the German Data Forum according to uniform and transparent standards (http://www.ratswd.de/en/data-infrastructure/rdc). The DEGS data set is freely accessible on application to interested scientists as de facto anonymized data for scientific secondary analysis. More detailed information on access, application forms and guidelines can be obtained from datennutzung@rki.de.

Code availability The code can be obtained from the corresponding author upon reasonable request after the permitted access to the DEGS data.

Authors' contributions $\mathrm{HK}$ and $\mathrm{AK}$ designed the study with substantial input from AR, JT, and CB. AR, JT, CS, CB, and AK contributed to the acquisition of data for the article. AK and HK designed the statistical analysis. HK conducted the statistical analysis. HK, AR, JT, HHK, $\mathrm{CB}$, and $\mathrm{AK}$ interpreted the data of the work. HK drafted the article with input from AR, JT, CS, HHK, CB, and AK. All authors critically revised the article for important intellectual content. All authors approved the final article.

Open Access This article is licensed under a Creative Commons Attribution-NonCommercial 4.0 International License, which permits any non-commercial use, sharing, adaptation, distribution and reproduction in any medium or format, as long as you give appropriate credit to the original author(s) and the source, provide a link to the Creative Commons licence, and indicate if changes were made. The images or other third party material in this article are included in the article's Creative Commons licence, unless indicated otherwise in a credit line to the 
material. If material is not included in the article's Creative Commons licence and your intended use is not permitted by statutory regulation or exceeds the permitted use, you will need to obtain permission directly from the copyright holder. To view a copy of this licence, visit http://creativecommons.org/licenses/by-nc/4.0/.

\section{References}

1. GBD 2017 Disease and Injury Incidence and Prevalence Collaborators. Global, regional, and national incidence, prevalence, and years lived with disability for 354 diseases and injuries for 195 countries and territories, 1990-2017: a systematic analysis for the Global Burden of Disease Study 2017. Lancet. 2017;392(10159):1789-858. https://doi.org/10.1016/s0140 $-6736(18) 32279-7$.

2. World Health Organization: Depression and other common mental disorders: global health estimates. In. Geneva, (2017).

3. Maske UE, Buttery AK, Beesdo-Baum K, Riedel-Heller S, Hapke U, Busch MA. Prevalence and correlates of DSM-IV-TR major depressive disorder, self-reported diagnosed depression and current depressive symptoms among adults in Germany. J Affect Disord. 2016;190:167-77. https://doi.org/10.1016/j.jad.2015.10.006.

4. Bretschneider J, Janitza S, Jacobi F, Thom J, Hapke U, Kurth $T$, Maske UE. Time trends in depression prevalence and health-related correlates: results from population-based surveys in Germany 1997-1999 vs. 2009-2012. BMC Psychiatry. 2018;18(1):394. https://doi.org/10.1186/s12888-018-1973-7.

5. König H, König H-H, Konnopka A. The excess costs of depression: a systematic review and meta-analysis. Epidemiol Psychiatr Sci. 2019. https://doi.org/10.1017/s2045796019000180.

6. Friedrich JO, Adhikari NK, Beyene J. Ratio of means for analyzing continuous outcomes in meta-analysis performed as well as mean difference methods. J Clin Epidemiol. 2011;64(5):556-64. https://doi.org/10.1016/j.jclinepi.2010.09.016.

7. Friedrich JO, Adhikari NK, Beyene J. The ratio of means method as an alternative to mean differences for analyzing continuous outcome variables in meta-analysis: a simulation study. BMC Med Res Methodol. 2008;8:32. https://doi. org/10.1186/1471-2288-8-32.

8. Fu R, Vandermeer BW, Shamliyan TA, O’Neil ME, Yazdi F, Fox SH, Morton SC. AHRQ methods for effective health care. Handling continuous outcomes in quantitative synthesis. In: Fu R, editor. Methods guide for effectiveness and comparative effectiveness reviews. Rockville: Agency for Healthcare Research and Quality (US); 2008.

9. Bock JO, Luppa M, Brettschneider C, Riedel-Heller S, Bickel H, Fuchs A, Gensichen J, Maier W, Mergenthal K, Schäfer I, Schön G, Weyerer S, Wiese B, van den Bussche H, Scherer M, König $\mathrm{HH}$. Impact of depression on health care utilization and costs among multimorbid patients-from the MultiCare Cohort Study. PLoS ONE. 2014;9(3):e91973. https://doi.org/10.1371/journ al.pone.0091973.

10. Bock JO, Brettschneider C, Weyerer S, Werle J, Wagner M, Maier W, Scherer M, Kaduszkiewicz H, Wiese B, Moor L, Stein J, Riedel-Heller SG, König HH. Excess health care costs of latelife depression - results of the AgeMooDe study. J Affect Disord. 2016;199:139-47. https://doi.org/10.1016/j.jad.2016.04.008.

11. Luppa M, Heinrich S, Matschinger H, Sandholzer H, Angermeyer MC, König HH, Riedel-Heller SG. Direct costs associated with depression in old age in Germany. J Affect Disord. 2008;105(13):195-204. https://doi.org/10.1016/j.jad.2007.05.008.

12. Hamre HJ, Witt CM, Glockmann A, Ziegler R, Kienle GS, Willich SN, Kiene H. Health costs in patients treated for depression, in patients with depressive symptoms treated for another chronic disorder, and in non-depressed patients: a 2-year prospective cohort study in anthroposophic outpatient settings. Eur J Health Econ. 2010;11(1):77-94. https://doi.org/10.1007/s1019 8-009-0203-0.

13. Stamm K, Reinhard I, Salize HJ. Long-term health insurance payments for depression in Germany - a secondary analysis of routine data. Neuropsychiatry. 2010;24(2):99-107.

14. Evans-Lacko S, Knapp M. Global patterns of workplace productivity for people with depression: absenteeism and presenteeism costs across eight diverse countries. Soc Psychiatry Psychiatr Epidemiol. 2016;51(11):1525-37. https://doi.org/10.1007/s0012 7-016-1278-4.

15. Krol M, Papenburg J, Koopmanschap M, Brouwer W. Do productivity costs matter? The impact of including productivity costs on the incremental costs of interventions targeted at depressive disorders. Pharmacoeconomics. 2011;29(7):601-19. https://doi. org/10.2165/11539970-000000000-00000.

16. Olesen J, Gustavsson A, Svensson M, Wittchen HU, Jonsson B. The economic cost of brain disorders in Europe. Eur J Neurol. 2012;19(1):155-62. https://doi.org/10.111 1/j.1468-1331.2011.03590.x.

17. Richardson RA, Keyes KM, Medina JT, Calvo E. Sociodemographic inequalities in depression among older adults: cross-sectional evidence from 18 countries. Lancet Psychiatry. 2020;7(8):673-81. https://doi.org/10.1016/s2215-0366(20)30151 $-6$.

18. Scheidt-Nave C, Kamtsiuris P, Gößwald A, Hölling H, Lange M, Busch AM, Dahm S, Dölle R, Ellert U, Fuchs J, Hapke U, Heidemann C, Knopf H, Laussmann D, Mensink BMG, Neuhauser H, Richter A, Sass A-C, Schaffrath-Rosario A, Stolzenberg H, Thamm M, Kurth B-M. German Health Interview and Examination Survey for Adults (DEGS)—design, objectives and implementation of the first data collection wave. BMC Public Health. 2012;12:730.

19. Kamtsiuris P, Lange M, Hoffmann R, Schaffrath Rosario A, Dahm S, Kuhnert R, Kurth BM. The first wave of the German Health Interview and Examination Survey for Adults (DEGS1): sample design, response, weighting and representativeness. Gesundheitsforsch Gesundheitssch. 2013;56(5-6):620-30. https://doi. org/10.1007/s00103-012-1650-9 (English version).

20. Gößwald A, Lange M, Dölle R, Hölling H. The first wave of the German Health Interview and Examination Survey for Adults (DEGS1): participant recruitment, fieldwork, and quality management. Bundesgesundheitsblatt Gesundheitsforschung Gesundheitsschutz. 2013;56(5-6):611-9.

21. Löwe B, Kroenke K, Herzog W, Gräfe K. Measuring depression outcome with a brief self-report instrument: sensitivity to change of the Patient Health Questionnaire (PHQ-9). J Affect Disord. 2004;81(1):61-6. https://doi.org/10.1016/S0165-0327(03)00198 $-8$.

22. Kroenke K, Spitzer RL, Williams JB. The PHQ-9: validity of a brief depression severity measure. J Gen Intern Med. 2001;16(9):606-13. https://doi.org/10.104 6/j.1525-1497.2001.016009606.x.

23. Chow W, Doane M, Sheehan J, Alphs L, Le H. Economic burden among patients with major depressive disorder: an analysis of healthcare resource use, work productivity, and direct and indirect costs by depression severity. Am J Manag Care Suppl Clin Brief. 2019.

24. McTernan WP, Dollard MF, LaMontagne AD. Depression in the workplace: an economic cost analysis of depression-related productivity loss attributable to job strain and bullying. Work Stress. 2013;27(4):321-38.

25. Wright DR, Katon WJ, Ludman E, McCauley E, Oliver M, Lindenbaum J, Richardson LP. Association of adolescent depressive symptoms with health care utilization and payer-incurred 
expenditures. Acad Pediatr. 2016;16(1):82-9. https://doi. org/10.1016/j.acap.2015.08.013.

26. Deutsche Rentenversicherung Bund: Statistik der Deutschen Rentenversicherung. Rehabilitation 2011. In, vol. Band 189. Würzburg, 2012.

27. Rattay P, Butschalowsky H, Rommel A, Prutz F, Jordan S, Nowossadeck E, Domanska O, Kamtsiuris P. Utilization of outpatient and inpatient health services in Germany: results of the German Health Interview and Examination Survey for Adults (DEGS1). Bundesgesundheitsblatt Gesundheitsforschung Gesundheitsschutz. 2013;56(5-6):832-44. https://doi.org/10.1007/s0010 3-013-1665-x.

28. Bock JO, Brettschneider C, Seidl H, Bowles D, Holle R, Greiner W, König HH. Calculation of standardised unit costs from a societal perspective for health economic evaluation. Gesundheitswesen. 2015;77(1):53-61. https://doi. org/10.1055/s-0034-1374621.

29. Rote Liste: Arzneimittelinformationen für Deutschland. Rote Liste Service GmbH, 2011.

30. Statistisches Bundesamt: Verdienste und Arbeitskosten. Arbeitskosten im Produzierenden Gewerbe und im Dienstleistungsbereich- Ergebnisse für Deutschland - 2008. In, vol. Fachserie $16 \mathrm{Heft}$ 1. Wiesbaden, 2014

31. Statistisches Bundesamt: Verdienste und Arbeitskosten. Arbeitnehmerverdienste. 4. Vierteljahr 2011. In, vol. Fachserie 16 Reihe 2.1. Wiesbaden, 2012

32. OECD: Key Short-Term Economic Indicators. Prices and Purchasing Power Parities. Consumer prices-Annual inflation. https:// stats.oecd.org/Index.aspx? ?ataSetCode=PRICES_CPI\#. 2020. Accessed 16 Nov 2020.

33. Luppa M, Giersdorf J, Riedel-Heller S, Prütz F, Rommel A. Frequent attenders in the German healthcare system: determinants of high utilization of primary care services. Results from the crosssectional German Health Interview and Examination Survey for Adults (DEGS). BMC Fam Pract. 2019.

34. Hoebel J, Rattay P, Prutz F, Rommel A, Lampert T. Socioeconomic status and use of outpatient medical care: the case of Germany. PLoS ONE. 2016;11(5):e0155982. https://doi.org/10.1371/ journal.pone.0155982.

35. Rommel A, Bretschneider J, Kroll LE, Prütz F, Thom J. The utilization of psychiatric and psychotherapeutic services in Germany-individual determinants and regional differences. J Health Monit. 2017;2(4):3-22. https://doi.org/10.17886/ rki-gbe-2017-122.2.

36. Rommel A, Kroll LE. Individual and regional determinants for physical therapy utilization in Germany: multilevel analysis of national survey data. Phys Ther. 2017;97(5):512-23. https://doi. org/10.1093/ptj/pzx022.

37. Rommel A, Saß A-C, Born S, Ellert U. Die gesundheitliche Lage von Menschen mit Migrationshintergrund und die Bedeutung des sozioökonomischen Status- Erste Ergebnisse der Studie zur Gesundheit Erwachsener in Deutschland (DEGS1). Bundesgesundheitsblatt Gesundheitsforschung Gesundheitsschutz. 2015;58(6):543-52.

38. Klein J, von dem Knesebeck O. Inequalities in health care utilization among migrants and non-migrants in Germany: a systematic review. Int J Equity Health. 2018;17(1):160. https://doi. org/10.1186/s12939-018-0876-Z.

39. Mack S, Jacobi F, Gerschler A, Strehle J, Höfler M, Busch MA, Maske UE, Hapke U, Seiffert I, Gaebel W, Zielasek J, Maier W, Wittchen H-U. Self-reported utilization of mental health services in the adult German population-evidence for unmet needs? Results of the DEGS1-Mental Health Module (DEGS1-MH). Int J Methods Psychiatr Res. 2014;23(3):289-303. https://doi. org/10.1002/mpr.1438.
40. Lampert T, Kroll LE, Muters S, Stolzenberg H. Messung des sozioökonomischen Status in der Studie "Gesundheit in Deutschland aktuell" (GEDA). Bundesgesundheitsblatt Gesundheitsforschung Gesundheitsschutz. 2013;56(1):131-43. https://doi.org/10.1007/ s00103-012-1583-3.

41. Saß A-C, Grüne B, Brettschneider A-K, Rommel A, Razum $\mathrm{O}$, Ellert U. Beteiligung von Menschen mit Migrationshintergrund an Gesundheitssurveys des Robert Koch-Instituts. Bundesgesundheitsblatt Gesundheitsforschung Gesundheitsschutz. 2015;58(6):533-42.

42. Dalgard, O.S., Dowrick, C., Lehtinen, V., Vazquez-Barquero, J.L., Casey, P., Wilkinson, G., Ayuso-Mateos, J.L., Page, H., Dunn, G., Group, O. Negative life events, social support and gender difference in depression: a multinational community survey with data from the ODIN study. Soc Psychiatry Psychiatr Epidemiol. 2006;41(6):444-51. https://doi.org/10.1007/s00127-006-0051-5.

43. Hainmueller J. Entropy balancing for causal effects: a multivariate reweighting method to produce balanced samples in observational studies. Political Anal. 2012;20(1):25-46.

44. Glick HA, Doshi JA, Sonnad SS, Polsky D. Economic evaluation in clinical trials. Oxford: OUP; 2010.

45. Hainmueller J, Xu Y. Ebalance: a stata package for entropy balancing. J Stat Softw. 2013;54(7):1.

46. Krug S, Jordan S, Mensink GB, Müters S, Finger J, Lampert T. Körperliche Aktivität. Ergebnisse der Studie zur Gesundheit Erwachsener in Deutschland (DEGS1). Bundesgesundheitsblatt Gesundheitsforschung Gesundheitsschutz. 2013;56(5-6):765-71.

47. Lampert T, Von Der Lippe E, Müters S. Verbreitung des Rauchens in der Erwachsenenbevölkerung in Deutschland. Ergebnisse der Studie zur Gesundheit Erwachsener in Deutschland (DEGS1). Bundesgesundheitsblatt Gesundheitsforschung Gesundheitsschutz. 2013;56(5-6):802-8.

48. Carstensen J, Andersson D, Andre M, Engstrom S, Magnusson $\mathrm{H}$, Borgquist LA. How does comorbidity influence healthcare costs? A population-based cross-sectional study of depression, back pain and osteoarthritis. BMJ Open. 2012;2(2):e000809. https ://doi.org/10.1136/bmjopen-2011-000809.

49. Carta MG, Hardoy MC, Kovess V, Dell'Osso L, Carpiniello B. Could health care costs for depression be decreased if the disorder were correctly diagnosed and treated? Soc Psychiatry Psychiatr Epidemiol. 2003;38(9):490-2. https://doi.org/10.1007/s0012 7-003-0662-z.

50. Chiu M, Lebenbaum M, Cheng J, de Oliveira C, Kurdyak P. The direct healthcare costs associated with psychological distress and major depression: a population-based cohort study in Ontario, Canada. PLoS ONE. 2017;12(9):e0184268. https://doi. org/10.1371/journal.pone.0184268.

51. Choi S, Lee S, Matejkowski J, Baek YM. The relationships among depression, physical health conditions and healthcare expenditures for younger and older Americans. J Ment Health. 2014;23(3):1405. https://doi.org/10.3109/09638237.2014.910643.

52. Druss BG, Rosenheck RA, Sledge WH. Health and disability costs of depressive illness in a major US corporation. Am J Psychiatry. 2000;157(8):1274-8. https://doi.org/10.1176/appi.ajp.157.8.1274.

53. Greenberg PE, Fournier AA, Sisitsky T, Pike CT, Kessler RC. The economic burden of adults with major depressive disorder in the United States (2005 and 2010). J Clin Psychiatry. 2015;76(2):15562. https://doi.org/10.4088/JCP.14m09298.

54. Hsieh CR, Qin X. Depression hurts, depression costs: the medical spending attributable to depression and depressive symptoms in China. Health Econ. 2018;27(3):525-44. https://doi.org/10.1002/ hec. 3604.

55. Trivedi DN, Lawrence LW, Blake SG, Rappaport HM, Feldhaus JB. Study of the economic burden of depression. J Pharm Financ Econ Policy. 2004;13(4):51-66. 
56. Fortin M, Bravo G, Hudon C, Vanasse A, Lapointe L. Prevalence of multimorbidity among adults seen in family practice. Ann Fam Med. 2005;3(3):223-8. https://doi.org/10.1370/afm.272.

57. Schäfer I, Hansen H, Schön G, Höfels S, Altiner A, Dahlhaus A, Gensichen J, Riedel-Heller S, Weyerer S, Blank WA, König H-H, von dem Knesebeck O, Wegscheider K, Scherer M, van den Bussche H, Wiese B. The influence of age, gender and socioeconomic status on multimorbidity patterns in primary care. First results from the multicare cohort study. BMC Health Serv Res. 2012;12(1):89. https://doi.org/10.1186/1472-6963-12-89.

58. Shvartzman P, Weiner Z, Vardy D, Friger M, Sherf M, Biderman A. Health services utilization by depressive patients identified by the MINI questionnaire in a primary care setting. Scand J Prim Health Care. 2005;23(1):18-25.

59. Gameroff MJ, Olfson M. Major depressive disorder, somatic pain, and health care costs in an urban primary care practice. J Clin Psychiatry. 2006;67(8):1232-9.

60. Ludvigsson M, Bernfort L, Marcusson J, Wressle E, Milberg A. Direct costs of very old persons with subsyndromal depression: a 5-year prospective study. Am J Geriatr Psychiatry. 2018. https:// doi.org/10.1016/j.jagp.2018.03.007.

61. Dreger S, Buck C, Bolte G. Material, psychosocial and sociodemographic determinants are associated with positive mental health in Europe: a cross-sectional study. BMJ Open. 2014;4(5):e005095. https://doi.org/10.1136/bmjopen-2014-005095.

62. Fryers T, Melzer D, Jenkins R, Brugha T. The distribution of the common mental disorders: social inequalities in Europe. Clin Pract Epidemiol Ment Health. 2005;1:14. https://doi. org/10.1186/1745-0179-1-14.

63. Lindert J, Priebe S, Penka S, Napo F, Schouler-Ocak M, Heinz A. Versorgung psychisch kranker Patienten mit
Migrationshintergrund. Psychother Psychosom Med Psychol. 2008;58(03/04):123-9. https://doi.org/10.1055/s-2008-1067360.

64. Schepker R, Eberding A, Toker M. Familiäre Bewältigungsstrategien: Bewältigungsstrategien und Umgang mit Verhaltensauffälligkeiten Jugendlicher in Familien aus der Türkei unter besonderer Berücksichtigung jugendpsychiatrischer Versorgung. 2005.

65. Grupp H, König HH, Konnopka A. Cost measurement of mental disorders in Germany. J Ment Health Policy Econ. 2014;17(1):3-8.

66. Steffen A, Thom J, Jacobi F, Holstiege J, Bätzing J. Trends in prevalence of depression in Germany between 2009 and 2017 based on nationwide ambulatory claims data. J Affect Disord. 2020;271:239-47. https://doi.org/10.1016/j.jad.2020.03.082.

67. Maske UE, Hapke U, Riedel-Heller SG, Busch MA, Kessler RC. Respondents' report of a clinician-diagnosed depression in health surveys: comparison with DSM-IV mental disorders in the general adult population in Germany. BMC Psychiatry. 2017;17(1):39. https://doi.org/10.1186/s12888-017-1203-8.

68. Drost RM, Paulus AT, Ruwaard D, Evers SM. Inter-sectoral costs and benefits of mental health prevention: towards a new classification scheme. J Ment Health Policy Econ. 2013;16(4):179-86.

69. Drost RM, van der Putten IM, Ruwaard D, Evers S, Paulus ATG. Conceptualizations of the societal perspective within economic evaluations: a systematic review. Int J Technol Assess Health Care. 2017;33(2):251-60. https://doi.org/10.1017/s026646231 7000526.

70. Fazel S, Wolf A, Chang Z, Larsson H, Goodwin GM, Lichtenstein P. Depression and violence: a Swedish population study. Lancet Psychiatry. 2015;2(3):224-32. https://doi.org/10.1016/s2215 -0366(14)00128-x. 